\title{
COALESCENCE IN CRITICAL AND SUBCRITICAL GALTON-WATSON BRANCHING PROCESSES
}

\author{
K. B. ATHREYA, ${ }^{*}$ Iowa State University
}

\begin{abstract}
In a Galton-Watson branching process that is not extinct by the $n$th generation and has at least two individuals, pick two individuals at random by simple random sampling without replacement. Trace their lines of descent back in time till they meet. Call that generation $X_{n}$ a pairwise coalescence time. Similarly, let $Y_{n}$ denote the coalescence time for the whole population of the $n$th generation conditioned on the event that it is not extinct. In this paper the distributions of $X_{n}$ and $Y_{n}$, and their limit behaviors as $n \rightarrow \infty$ are discussed for both the critical and subcritical cases.
\end{abstract}

Keywords: Branching process; coalescence; critical; subcritical

2010 Mathematics Subject Classification: Primary 60J80

Secondary 60F10

\section{Introduction}

Let $\left\{p_{j}\right\}_{j \geq 0}$ be a probability distribution on the nonnegative integers $\mathbb{N}^{+} \equiv\{0,1,2, \ldots\}$. Let $\left\{\xi_{n, i}: i \geq 1, n \geq 0\right\}$ be a doubly infinite family of independent random variables with distribution $\left\{p_{j}\right\}_{j \geq 0}$. Let $\left\{Z_{n}\right\}_{n \geq 0}$ be a sequence of random variables defined by the stochastic recurrence relation $1 \leq Z_{0}<\infty$ nonrandom with values in $\mathbb{N}^{+}$and, for $n=0,1,2, \ldots$,

$$
Z_{n+1}= \begin{cases}\sum_{i=1}^{Z_{n}} \xi_{n, i} & \text { if } Z_{n}>0, \\ 0 & \text { if } Z_{n}=0 .\end{cases}
$$

Then the sequence $\left\{Z_{n}\right\}_{n \geq 0}$ is called the population size sequence of a Galton-Watson branching process with offspring distribution $\left\{p_{j}\right\}_{j \geq 0}$ and initiated size $Z_{0}$. (See [2].)

Here $Z_{n}$ is the size of the $n$th generation and, for any $n$ and $i, \xi_{n, i}$ denotes the number of offspring of the $i$ th individual in the $n$th generation. If $\mathbb{T}$ denotes the full family tree generated, every individual in $\mathbb{T}$ can be identified by a finite string $\left(i_{0}, i_{1}, \ldots, i_{n}\right)$, meaning that this individual is in the $n$th generation and is the $i_{n}$ th offspring of individual $\left(i_{0}, i_{1}, \ldots, i_{n-1}\right)$ in the $(n-1)$ th generation. For any $0 \leq k<n$, individual $\left(i_{0}, i_{1}, \ldots, i_{k}\right)$ is a common ancestor of all individuals $\left(i_{0}, i_{1}, \ldots, i_{k}, i_{k+1}, \ldots, i_{n}\right)$, where $i_{k+1}, i_{k+2}, \ldots, i_{n}$ are any positive integers.

The coalescence time of two individuals of any generation is the generation number of their last common ancestor. Clearly, every ancestor of this common ancestor is also a common ancestor.

Now, for each $n \geq 1$, consider the event $A_{n} \equiv\left\{Z_{n} \geq 2\right\}$. For a family tree that is in $A_{n}$, choose two individuals at random from those in the $n$th generation by simple random sampling

Received 17 September 2010; revision received 18 January 2012.

* Postal address: Departments of Mathematics and Statistics, Iowa State University, Ames, Iowa, 50011, USA.

Email address: kba@iastate.edu 
without replacement. Now trace their lines of descent back in time till they meet at their last common ancestor. Call the generation of the last common ancestor of those randomly chosen two individuals a pairwise coalescence time $X_{n}$.

Next, for each $n \geq 1$, consider the event $B_{n} \equiv\left\{Z_{n} \geq 1\right\}$. For a family tree $\mathbb{T}$ in $B_{n}$, trace the lines of descent of all the individuals in the $n$th generation till they meet. Call that generation number $Y_{n}$ the total coalescence time.

In this paper we determine the limit behavior of the distribution of $X_{n}$ conditioned on $A_{n}$ and that of $Y_{n}$ conditioned on $B_{n}$ as $n \rightarrow \infty$ in the nonsupercritical cases, i.e. when $\left\{p_{j}\right\}_{j \geq 0}$ satisfies the condition $0<m \equiv \sum_{j=1}^{\infty} j p_{j} \leq 1$.

The supercritical case, i.e. $m>1$, including the explosion case, $m=\infty$, is treated in [1]. See Remark 2.2 in the next section.

\section{Main results}

Let $\left\{Z_{n}, A_{n}, B_{n}, X_{n}, Y_{n},\left\{p_{j}\right\}_{j \geq 0}, m, \mathbb{T}\right\}$ be as in the introduction.

Theorem 2.1. (Critical case.) Let $m \equiv \sum_{j=1}^{\infty} j p_{j}=1, p_{1}<1$, and $\sigma^{2} \equiv \sum_{j=1}^{\infty} j^{2} p_{j}-$ $1<\infty$. Then the following statements hold.

(i) For $0<u<1$,

$$
\lim _{n \rightarrow \infty} \mathrm{P}\left(X_{n}<n u \mid Z_{n} \geq 2\right) \equiv H(u)
$$

exists and equals $1-\mathrm{E} \phi\left(N_{u}\right)$, where $N_{u}$ is a positive integer-valued random variable with a geometric distribution $\mathrm{P}\left(N_{u}=k\right)=(1-u) u^{k-1}, k \geq 1$, and, for $j \geq 1$,

$$
\phi(j)=\mathrm{E}\left(\frac{\sum_{i=1}^{j} \eta_{i}^{2}}{\left(\sum_{i=1}^{j} \eta_{i}\right)^{2}}\right)
$$

where $\left\{\eta_{i}\right\}_{i \geq 1}$ are independent and identically distributed (i.i.d.) exponential random variables with mean 1 .

Furthermore, $H(\cdot)$ is an absolutely continuous cumulative distribution function on $[0,1]$ with $H(0+)=0$ and $H(1-)=1$.

(ii) For $0<u<1$,

$$
\lim _{n \rightarrow \infty} \mathrm{P}\left(Y_{n}>n u \mid Z_{n} \geq 1\right)=1-u .
$$

Remark 2.1. Theorem 2.1(ii) is a known result proved by Zubkov [6].

Theorem 2.2. (Subcritical case.) Let $0<m \equiv \sum_{j=1}^{\infty} j p_{j}<1$. Then the following statements hold.

(i) For $k \geq 1$,

$$
\lim _{n \rightarrow \infty} \mathrm{P}\left(n-X_{n}>k \mid Z_{n} \geq 2\right)=\frac{\mathrm{E} \phi_{k}(Y)}{\mathrm{E} \psi_{k}(Y)} \equiv \pi_{k}, \quad s a y,
$$

where

$$
\phi_{k}(j)=\mathrm{E}\left(\frac{\sum_{i_{1} \neq i_{2}=1}^{j} Z_{k, i_{1}} Z_{k, i_{2}}}{\left(\sum_{i=1}^{j} Z_{k, i}\right)\left(\sum_{i=1}^{j} Z_{k, i}-1\right)} \mathbf{1}\left(\sum_{i=1}^{j} Z_{k, i} \geq 1\right)\right),
$$




$$
\psi_{k}(j)=\mathrm{P}\left(\sum_{i=1}^{j} Z_{k, i} \geq 2\right),
$$

$\left\{Z_{r, i}: r \geq 0\right\}, i=1,2, \ldots$, are i.i.d. copies of a Galton-Watson branching process $\left\{Z_{r}: r \geq 0\right\}$ with $Z_{0}=1$ and the given offspring distribution $\left\{p_{j}\right\}_{j \geq 0}$, and $Y$ is a random variable with distribution $\left\{b_{j}\right\}_{j \geq 1}$, where $b_{j} \equiv \lim _{n \rightarrow \infty} \mathrm{P}\left(Z_{n}=j \mid Z_{n}>0\right.$, $Z_{0}=1$ ), which exists (as asserted in Theorem 3.3 below).

Furthermore, if $\sum_{j=1}^{\infty} j \log j p_{j}<\infty$ then $\lim _{k \uparrow \infty} \pi_{k}=0$ and, hence, $n-X_{n}$ conditioned on $Z_{n} \geq 2$ converges to a proper distribution on $\{1,2, \ldots\}$.

(ii) For $k \geq 1, \lim _{n \rightarrow \infty} \mathrm{P}\left(n-Y_{n}>k \mid Z_{n} \geq 1\right) \equiv \tilde{\pi}_{k}$ exists and equals

$$
\mathrm{E}\left(\frac{1-q_{k}^{Y}}{m^{k}}\right)-\mathrm{E}\left(\frac{Y q_{k}^{Y-1}\left(1-q_{k}\right)}{m^{k}}\right),
$$

where $Y$ is a random variable with distribution

$$
\mathrm{P}(Y=j)=b_{j}=\lim _{n \rightarrow \infty} \mathrm{P}\left(Z_{n}=j \mid Z_{n}>0, Z_{0}=1\right)
$$

and $q_{k}=\mathrm{P}\left(Z_{k}=0 \mid Z_{0}=1\right)$.

Furthermore, if $\sum_{j=1}^{\infty} j \log j p_{j}<\infty$ then

$$
\lim _{k \rightarrow \infty} \tilde{\pi}_{k}=0
$$

That is, $n-Y_{n}$ conditioned on $\left\{Z_{n}>0\right\}$ converges in distribution as $n \rightarrow \infty$ to a proper distribution on $\{1,2, \ldots\}$.

Remark 2.2. Theorem 2.2 provides a sharp contrast to Theorem 2.1. In the subcritical case, the coalescence times $X_{n}$ and $Y_{n}$ are close to the present time $n$, whereas in the critical case they are both of the order of magnitude $n$ and, hence, could be a long time before the present but also a long time from the initial ancestor. In the supercritical case with $1<m \equiv \sum_{j=1}^{\infty} j p_{j}<\infty$, it was shown in [1] that $X_{n}$ converges in distribution, i.e. coalescence takes place close to the ancestor, with the same being true for $Y_{n}$. Thus, we have a trichotomy based on the value of the offspring mean $m$. For $1<m<\infty$, the coalescence is close to the ancestor, i.e. beginning of the tree. For $m=1$, it is of the order $n$, neither close to the ancestor nor close to the present. For $0<m<1$, it is close to the present. Another unexpected result is the following. It turns out that, when $m=\infty$ and $\left\{p_{j}\right\}_{j \geq 0}$ is in the domain of attraction of a stable law of order $\alpha, 0<\alpha<1$, the coalescence time $X_{n}$ is close to the present and, in fact, $n-X_{n}$ converges in distribution to a proper distribution (as in the subcritical case). See [1] for details on this case.

Remark 2.3. The referee pointed out that Theorem 2.1 could also be proved using the excursion representation of the limiting continuum random trees (see [5]). According to the referee,

in the limiting continuum tree conditioned to reach height 1 (which is known to have an exponentially distributed width at height 1 ) there is a geometric number $N_{u}$ of subtrees whose MRCAs lived before time $u<1$ and whose widths at time 1 are constant multiples of the $\eta_{i}$ s in Theorem 2.1. Further, the event that two individuals sampled randomly at time 1 have a 'coalescence time' earlier than $u$ is just the event that these individuals do not belong to the same subtree. 
Remark 2.4. The referee also pointed out that the $L \log L$ condition in Theorem 2.2 can be dropped for the case $Z_{0}=1$ using the results of Geiger [3], and raised the question of whether Theorem 2.1 can be proved for a random $Z_{0}$ with just $\mathrm{E} Z_{0}<\infty$ but without the $L \log L$ condition. The present author hopes to investigate these issues in the near future.

\section{Some preliminary results}

In this section we collect some well-known results, and present some new results on GaltonWatson branching processes in the critical and subcritical cases.

Theorem 3.1. (Critical case.) Let $m=1, p_{1}<1$, and $0<\sigma^{2} \equiv \sum_{j=1}^{\infty} j^{2} p_{j}-1<\infty$. Let $Z_{0}=k<\infty$. Then, as $n \rightarrow \infty$,

(i) $n \mathrm{P}\left(Z_{n}>0\right) \rightarrow 2 / \sigma^{2}$;

(ii) $\mathrm{P}\left(Z_{n} / n>u \mid Z_{n}>0\right) \rightarrow \mathrm{e}^{-2 u / \sigma^{2}}, 0<u<\infty$.

For a proof, see [2, p. 20].

Let $\left\{Z_{j, i}^{(k)}: j \geq 0\right\}$ be the Galton-Watson branching process initiated by the $i$ th individual in the $k$ th generation, $1 \leq i \leq Z_{k}$.

Theorem 3.2. Under the hypothesis and notation of Theorem 3.1, consider the point process

$$
V_{n} \equiv\left\{\frac{Z_{n-k, i}^{(k)}}{n-k}: 1 \leq i \leq Z_{k}, Z_{n-k, i}^{(k)}>0\right\}
$$

on the event $B_{n} \equiv\left\{Z_{n}>0\right\}$. Then, as $n \rightarrow \infty, k \rightarrow \infty$, and $k / n \rightarrow u, 0<u<1$, conditioned on the event $B_{n}$, the point process sequence $V_{n}$ converges to $V \equiv\left\{\eta_{i}: 1 \leq i \leq N_{u}\right\}$, where $\left\{\eta_{i}\right\}_{i \geq 1}$ are i.i.d. exponential random variables with mean $\sigma^{2} / 2$ and $N_{u}$ is independent of $\left\{\eta_{i}\right\}_{i \geq 1}$ with distribution $\mathrm{P}\left(N_{u}=k\right)=(1-u) u^{k-1}, k \geq 1$.

Proof. Let $f: \mathbb{R}^{+} \equiv[0, \infty) \rightarrow \mathbb{R}^{+}$be a bounded continuous function. Let, for $n \geq 1$ and $1 \leq k \leq n$,

$$
Y_{n, k}=\exp \left(-s \sum_{i=1}^{Z_{k}} f\left(\frac{Z_{n-k, i}^{(k)}}{n-k}\right) \mathbf{1}\left(Z_{n-k, i}^{(k)}>0\right)\right)
$$

Then

$$
\begin{aligned}
\mathrm{E}\left(Y_{n, k}\right. & \left.\mathbf{1}\left(Z_{n}>0\right) \mid Z_{j}: j \leq k\right) \\
\quad= & \mathrm{E}\left(Y_{n, k} \mathbf{1}\left(Z_{k}>0\right) \mathbf{1}\left(Z_{n}>0\right) \mid Z_{k}\right) \quad \text { (by the Markov property) } \\
\quad= & \mathrm{E}\left(Y_{n, k} \mathbf{1}\left(Z_{k}>0\right) \mid Z_{k}\right)-\mathrm{E}\left(Y_{n, k} \mathbf{1}\left(Z_{k}>0\right) \mathbf{1}\left(Z_{n}=0\right) \mid Z_{k}\right) \\
\quad= & \left(g_{n-k}(s)\right)^{Z_{k}} \mathbf{1}\left(Z_{k}>0\right)-q_{n-k}^{Z_{k}} \mathbf{1}\left(Z_{k}>0\right),
\end{aligned}
$$

where

$$
g_{j}(s)=\mathrm{E}\left(\exp \left(-s f\left(\frac{Z_{j}}{j}\right) \mathbf{1}\left(Z_{j}>0\right)\right) \mid Z_{0}=1\right)
$$

and

$$
q_{j}=\mathrm{P}\left(Z_{j}=0 \mid Z_{0}=1\right), \quad j \geq 1 .
$$


Now,

$$
g_{j}(s)=q_{j}+\left(1-q_{j}\right) \mathrm{E}\left(\exp \left(-s f\left(\frac{Z_{j}}{j}\right)\right) \mid Z_{j}>0, Z_{0}=1\right) .
$$

From Theorem 3.1(i), under the hypothesis of Theorem 2.1, as $j \rightarrow \infty$,

$$
j\left(1-q_{j}\right) \rightarrow \frac{2}{\sigma^{2}}
$$

and

$$
\frac{Z_{j}}{j} \mid Z_{j}>0 \stackrel{\mathrm{D}}{\rightarrow} \text { an exponential distribution with mean } \frac{2}{\sigma^{2}} .
$$

Clearly,

$$
g_{j}(s)=\left(1+\left(1-q_{j}\right)\left(\tilde{g}_{j}(s)-1\right)\right)
$$

where

$$
\tilde{g}_{j}(s)=\mathrm{E}\left(\exp \left(-s f\left(\frac{Z_{j}}{j}\right)\right) \mid Z_{j}>0, Z_{0}=1\right) .
$$

Since $f$ is bounded and continuous, it follows from Theorem 3.1 that, as $j \rightarrow \infty$,

$$
\tilde{g}_{j}(s) \rightarrow \frac{2}{\sigma^{2}} \int_{0}^{\infty} \mathrm{e}^{-s f(x)} \mathrm{e}^{-2 x / \sigma^{2}} \mathrm{~d} x \equiv \tilde{g}(s), \quad \text { say, }
$$

implying that $\left(g_{j}(s)\right)^{j} \rightarrow \mathrm{e}^{2(\tilde{g}(s)-1) / \sigma^{2}}$.

Now, let $n \rightarrow \infty, k \rightarrow \infty$, and $k / n \rightarrow u, 0<u<1$. Then

$$
\begin{aligned}
\lim _{n \rightarrow \infty} & \mathrm{E}\left(\left(g_{n-k}(s)\right)^{Z_{k}} \mathbf{1}\left(Z_{k}>0\right) \mid Z_{k}>0\right) \\
& =\lim _{n \rightarrow \infty} \mathrm{E}\left(\left(\left(g_{n-k}(s)\right)^{n-k}\right)^{\left(Z_{k} / k\right) k /(n-k)} \mid Z_{k}>0\right) \\
& =\frac{2}{\sigma^{2}} \int_{0}^{\infty} \exp \left(\frac{2}{\sigma^{2}}(\tilde{g}(s)-1)\left(\frac{u}{1-u}\right) x\right) \mathrm{e}^{-2 x / \sigma^{2}} \mathrm{~d} x \\
& =\frac{2 / \sigma^{2}}{\left(2 / \sigma^{2}\right)(1-(\tilde{g}(s)-1) u /(1-u))} \\
& =\frac{1-u}{1-u \tilde{g}(s)} .
\end{aligned}
$$

Similarly,

$$
\mathrm{E}\left(q_{n-k}^{Z_{k}} \mid Z_{k}>0\right) \rightarrow 1-u
$$

Thus,

$$
\begin{aligned}
\mathrm{E}\left(Y_{n, k} \mid Z_{n}>0\right) & =\mathrm{E}\left(\mathrm{E}\left(Y_{n, k} \mathbf{1}\left(Z_{n}>0\right) \mid Z_{j}: j \leq k\right) \mid Z_{n}>0\right) \\
& \rightarrow \frac{1-u}{u}\left(\frac{1}{1-u \tilde{g}(s)}-1\right) \\
& =\frac{(1-u) u \tilde{g}(s)}{u(1-u \tilde{g}(s))} \\
& =(1-u) \sum_{j=0}^{\infty} u^{j}(\tilde{g}(s))^{j+1} \\
& =\sum_{j=1}^{\infty}(1-u) u^{j-1}(\tilde{g}(s))^{j} .
\end{aligned}
$$


This equals $\mathrm{E}\left(\exp \left(-s \sum_{i=1}^{N_{u}} f\left(\eta_{i}\right)\right)\right)$, where $\left\{\eta_{i}\right\}_{i \geq 1}$ and $N_{u}$ are as in the statement of Theorem 3.1. Now, by the continuity theorem for point processes (see [4]), Theorem 3.2 follows.

Theorem 3.3. (Subcritical case.) Let $0<m \equiv \sum_{j=1}^{\infty} j p_{j}<1$ and $Z_{0}=1$. Then, for $j \geq 1$, the following statements hold.

(i) $\lim _{n \rightarrow \infty} \mathrm{P}\left(Z_{n}=j \mid Z_{n}>0\right) \equiv b_{j}$ exists and $\sum_{j=1}^{\infty} b_{j}=1$. Furthermore, $B(s) \equiv$ $\sum_{j=1}^{\infty} b_{j} s^{j}, 0 \leq s \leq 1$, is the unique solution of the functional equation

$$
B(f(s))=m B(s)+(1-s), \quad 0 \leq s \leq 1,
$$

in the class of probability generating functions vanishing at 0 .

(ii) $\sum_{j=1}^{\infty} j b_{j}<\infty$ if and only if $\sum_{j=1}^{\infty} j \log j p_{j}<\infty$.

(iii) If $\sum_{j=1}^{\infty} j \log j p_{j}<\infty$ then

$$
\lim _{n \rightarrow \infty} \frac{\mathrm{P}\left(Z_{n}>0\right)}{m^{n}}=\frac{1}{\sum_{j=1}^{\infty} j b_{j}}>0 .
$$

For a proof, see [2, pp. 16, 18, 40].

The next result extends the above to random initial $Z_{0}$.

Theorem 3.4. Let $0<m<1$ and $Z_{0}$ be a random variable such that $\mathrm{P}\left(Z_{0} \geq 1\right)=1$ and E $Z_{0}<\infty$. Then,

(i) for $j \geq 1$,

$$
\lim _{n \rightarrow \infty} \mathrm{P}\left(Z_{n}=j \mid Z_{n}>0\right)=b_{j},
$$

where $\left\{b_{j}\right\}_{j \geq 1}$ is as in Theorem 3.3(i);

(ii) if $\sum_{j=1}^{\infty} j \log j p_{j}<\infty, \lim _{n \rightarrow \infty} \mathrm{P}\left(Z_{n}>0\right) / m^{n}$ exists in $(0, \infty)$ and equals $\mathrm{E} Z_{0} / \sum_{j=1}^{\infty} j b_{j}$.

Proof. For $0 \leq s \leq 1$,

$$
\mathrm{E}\left(s^{Z_{n}} \mid Z_{0}\right)=\left(f_{n}(s)\right)^{Z_{0}}
$$

where $f_{n}(s)=\mathrm{E}\left(s^{Z_{n}} \mid Z_{0}=1\right)$. So,

$$
\begin{aligned}
\mathrm{E}\left(s^{Z_{n}} \mid Z_{n}>0\right) & =\frac{\mathrm{E}\left(s^{Z_{n}} \mathbf{1}\left(Z_{n}>0\right)\right)}{\mathrm{P}\left(Z_{n}>0\right)} \\
& =\frac{\mathrm{E}\left(s^{Z_{n}}\right)-\mathrm{E}\left(s^{Z_{n}} \mathbf{1}\left(Z_{n}=0\right)\right)}{\mathrm{P}\left(Z_{n}>0\right)} \\
& =\frac{\mathrm{E}\left(f_{n}(s)\right)^{Z_{0}}-\mathrm{E}\left(f_{n}(0)\right)^{Z_{0}}}{1-\mathrm{E}\left(f_{n}(0)\right)^{Z_{0}}} \\
& =1-\frac{\mathrm{E}\left(1-\left(f_{n}(s)\right)^{Z_{0}}\right)}{\mathrm{E}\left(1-\left(f_{n}(0)\right)^{Z_{0}}\right)}
\end{aligned}
$$

By the monotone convergence theorem,

$$
\lim _{n \rightarrow \infty} \mathrm{E}\left(\frac{1-\left(f_{n}(s)\right)^{Z_{0}}}{1-f_{n}(s)}\right)=\mathrm{E} Z_{0} \quad \text { for } 0 \leq s<1 .
$$


Also, by Theorem 3.3(i),

$$
\lim _{n \rightarrow \infty} \frac{f_{n}(s)-f_{n}(0)}{1-f_{n}(0)}=B(s) \quad \text { for } 0 \leq s<1,
$$

and, hence,

$$
\lim _{n \rightarrow \infty} \frac{1-f_{n}(s)}{1-f_{n}(0)}=1-B(s) \quad \text { for } 0 \leq s<1 .
$$

Since, by hypothesis, $0<\mathrm{E} Z_{0}<\infty$,

$$
\lim _{n \rightarrow \infty} \mathrm{E}\left(s^{Z_{n}} \mid Z_{n}>0\right)=\lim _{n \rightarrow \infty}\left(1-(1-B(s)) \frac{\mathrm{E} Z_{0}}{\mathrm{E} Z_{0}}\right)=B(s),
$$

proving (i).

Next,

$$
\frac{\mathrm{P}\left(Z_{n}>0\right)}{m^{n}}=\frac{\mathrm{E}\left(1-\left(f_{n}(0)\right)^{Z_{0}}\right)}{m^{n}}=\frac{1-f_{n}(0)}{m^{n}} \mathrm{E}\left(\sum_{j=0}^{Z_{0}-1}\left(f_{n}(0)\right)^{j}\right) .
$$

By Theorem 3.3(iii), under the hypothesis that $\sum_{j=1}^{\infty} j \log j p_{j}<\infty$, the first term on the righthand side above converges to $1 / \sum_{j=1}^{\infty} j b_{j}$, which is positive and finite. By the monotone convergence theorem, the second term on the right-hand side above converges to $\mathrm{E} Z_{0}$. This proves (ii).

Corollary 3.1. Let $0<m<1$ and $Z_{0}$ be a finite nonrandom integer greater than or equal to 1 . Then, for $j \geq 1$,

$$
\lim _{n \rightarrow \infty} \mathrm{P}\left(Z_{n}=j \mid Z_{n}>0\right)=b_{j}
$$

where $\left\{b_{j}\right\}_{j \geq 1}$ is as in Theorem 3.3(i).

\section{Proofs of the main results}

\subsection{Proof of Theorem 2.1}

(i) The event $\left\{X_{n}<k\right\}$ for $1 \leq k \leq n$ occurs if and only if the two randomly chosen individuals from the $n$th generation come from the $(n-k)$ th generation of the trees initiated by two distinct individuals of the $k$ th generation. Also, the total number of choices of the two individuals from the $n$th generation is $Z_{n}\left(Z_{n}-1\right)$. Thus, for $n \geq 1$ and $k \geq 1$,

$$
\mathrm{P}\left(X_{n}<k \mid Z_{n} \geq 2\right)=\mathrm{E}\left(\frac{\sum_{i_{1} \neq i_{2}=1}^{Z_{k}} Z_{n-k, i_{1}}^{(k)} Z_{n-k, i_{2}}^{(k)}}{Z_{n}\left(Z_{n}-1\right)} \mid Z_{n} \geq 2\right) .
$$

Since

$$
\sum_{i_{1} \neq i_{2}=1}^{Z_{k}} Z_{n-k, i_{1}}^{(k)} Z_{n-k, i_{2}}^{(k)}=\left(\sum_{i=1}^{Z_{k}} Z_{n-k, i}^{(k)}\right)^{2}-\sum_{i=1}^{Z_{k}}\left(Z_{n-k, i}^{(k)}\right)^{2}
$$


it suffices to show that, as $k \rightarrow \infty, n \rightarrow \infty$, and $k / n \rightarrow u, 0<u<1$, the quantity

$$
\mathrm{E}\left(\frac{\sum_{i=1}^{Z_{k}}\left(Z_{n-k, i}^{(k)}\right)^{2}}{\left(\sum_{i=1}^{Z_{k}} Z_{n-k, i}^{(k)}\right)^{2}} \mid Z_{n} \geq 2\right)
$$

converges to $1-H(u)$ defined in (i).

Next, by Theorem 3.1(ii), $Z_{n} / n$ conditioned on $Z_{n}>0$ converges in distribution to an exponential distribution with mean $2 / \sigma^{2}$; thus, it follows that

$$
\mathrm{P}\left(Z_{n} \geq 2 \mid Z_{n}>0\right) \rightarrow 1 \quad \text { as } n \rightarrow \infty .
$$

Also, the quantity

$$
\frac{\sum_{i=1}^{Z_{k}}\left(Z_{n-k, i}^{(k)}\right)^{2}}{\left(\sum_{i=1}^{Z_{k}} Z_{n-k, i}^{(k)}\right)^{2}}=\frac{\sum_{i \in J_{k}}\left(Z_{n-k, i}^{(k)} /(n-k)\right)^{2}}{\left(\sum_{i \in J_{k}} Z_{n-k, i}^{(k)} /(n-k)\right)^{2}},
$$

where $J_{k} \equiv\left\{i: Z_{n-k, i}^{(k)}>0\right\}, 0 \leq k \leq n$, is a continuous functional in the appropriate topology of point processes on $\mathbb{R}^{+}$of the point process $V_{n}$ conditioned on the event $\left\{Z_{n}>0\right\}$. Thus, by the weak convergence result in Theorem 3.2, as $n \rightarrow \infty, k \rightarrow \infty$, and $k / n \rightarrow u, 0<u<1$,

$$
\mathrm{E}\left(\frac{\sum_{i \in J_{k}}\left(Z_{n-k, i}^{(k)}\right)^{2}}{\left(\sum_{i \in J_{k}} Z_{n-k, i}^{(k)}\right)^{2}} \mid Z_{n} \geq 2\right) \rightarrow \mathrm{E}\left(\frac{\sum_{i=1}^{N_{u}} \eta_{i}^{2}}{\left(\sum_{i=1}^{N_{u}} \eta_{i}\right)^{2}}\right),
$$

where $\left\{\eta_{i}\right\}_{i \geq 1}$ and $N_{u}$ are as in Theorem 3.2. This proves the convergence part of Theorem 2.1(i).

Next, since $\mathrm{E} \eta_{1}^{2}<\infty$ and $\mathrm{E} \eta_{1}>0$, by the strong law,

$$
\frac{\sum_{i=1}^{j} \eta_{i}^{2}}{\left(\sum_{i=1}^{j} \eta_{i}\right)^{2}} \rightarrow 0 \quad \text { with probability } 1 \text { as } j \rightarrow \infty .
$$

Now, by the bounded convergence theorem, $\phi(j) \rightarrow 0$ as $j \rightarrow \infty$. Also, as $u \uparrow 1, N_{u} \rightarrow \infty$ in distribution and, thus, $H(u) \uparrow 1$ as $u \uparrow 1$. Since $H(u)=1-\sum_{j=1}^{\infty} \phi(j)(1-u) u^{j-1}$, $H(\cdot)$ is an absolutely continuous cumulative distribution function on $[0,1]$ with $H(0+)=0$ and $H(1-)=1$. Thus, the proof of Theorem 2.1(i) is complete.

(ii) The event $\left\{Y_{n} \geq k\right\}$ for $1 \leq k \leq n$ conditioned on $\left\{Z_{n}>0\right\}$ occurs if and only if all the $Z_{n}$ individuals of the $n$th generation come from the $(n-k)$ th generation of a branching process initiated by exactly one individual of the $k$ th generation, i.e. $Z_{n-k, i}^{(k)}=0$ for all but one $i$, $1 \leq i \leq Z_{k}$, and $Z_{k}>0$. This yields

$$
\begin{aligned}
\mathrm{P}\left(Y_{n}\right. & \left.\geq k \mid Z_{n} \geq 1\right) \\
& =\frac{\mathrm{E}\left(Z_{n-k, i}^{(k)}=0 \text { for all but one } i, 1 \leq i \leq Z_{k}, \text { and } Z_{k}>0\right)}{\mathrm{P}\left(Z_{n}>0\right)} \\
& =\mathrm{E}\left(Z_{k} q_{n-k}^{Z_{k}-1}\left(1-q_{n-k}\right) \mid Z_{k}>0\right) \frac{\mathrm{P}\left(Z_{k}>0\right)}{\mathrm{P}\left(Z_{n}>0\right)} \quad\left(\text { where } q_{n}=\mathrm{P}\left(Z_{n}>0 \mid Z_{0}=1\right)\right) \\
& =\mathrm{E}\left(\frac{Z_{k}}{k} q_{n-k}^{Z_{k}-1} \frac{k}{n-k}(n-k)\left(1-q_{n-k}\right) \mid Z_{k}>0\right) \frac{\mathrm{P}\left(Z_{k}>0\right)}{\mathrm{P}\left(Z_{n}>0\right)} .
\end{aligned}
$$


Now, as $n \rightarrow \infty, k \rightarrow \infty$ and $k / n \rightarrow u, 0<u<1$, by Theorem 3.1,

$$
(n-k)\left(1-q_{n-k}\right) \rightarrow \frac{2}{\sigma^{2}}, \quad q_{n-k}^{n-k}=\left(1-\frac{(n-k)\left(1-q_{n-k}\right)}{n-k}\right)^{n-k} \rightarrow \mathrm{e}^{-2 / \sigma^{2}},
$$

and

$$
\frac{Z_{k}-1}{n-k}=\frac{Z_{k}-1}{k} \frac{k}{n-k},
$$

conditioned on $Z_{k}>0$, converges to $u /(1-u)$ times an exponential random variable with mean $\sigma^{2} / 2$. Also,

$$
\frac{\mathrm{P}\left(Z_{k}>0\right)}{\mathrm{P}\left(Z_{n}>0\right)}=\frac{k \mathrm{P}\left(Z_{k}>0\right)}{n \mathrm{P}\left(Z_{n}>0\right)} \frac{n}{k} \rightarrow \frac{1}{u}
$$

Thus, as $n \rightarrow \infty$,

$$
\mathrm{P}\left(Y_{n} \geq k \mid Z_{n} \geq 1\right) \rightarrow \frac{1}{u} \mathrm{E}\left(\frac{\sigma^{2}}{2} \eta \exp \left(-\frac{2}{\sigma^{2}} \frac{u}{1-u} \frac{\sigma^{2}}{2} \eta\right) \frac{2}{\sigma^{2}} \frac{u}{1-u}\right),
$$

where $\eta$ is an exponential random variable with mean 1 .

The above limit equals

$$
\frac{1}{1-u} \mathrm{E}\left(\eta \exp \left(-\left(\frac{u}{1-u}\right) \eta\right)\right)=\frac{1}{1-u}(1-u)^{2}=1-u
$$

since, for any $\theta>0$,

$$
\mathrm{E}\left(\eta \mathrm{e}^{-\theta \eta}\right)=\int_{0}^{\infty} x \mathrm{e}^{-\theta x} \mathrm{e}^{-x} \mathrm{~d} x=\frac{1}{(1+\theta)^{2}} .
$$

This proves Theorem 2.1(ii).

\subsection{Proof of Theorem 2.2}

(i) For $0 \leq k<n<\infty$,

$$
\mathrm{P}\left(n-X_{n}>k \mid Z_{n} \geq 2\right)=\frac{\mathrm{P}\left(X_{n}<n-k, Z_{n} \geq 2\right)}{\mathrm{P}\left(Z_{n} \geq 2\right)}=\frac{a_{n}}{c_{n}}, \quad \text { say. }
$$

Now,

$$
\begin{aligned}
a_{n} & =\mathrm{E}\left(\frac{\sum_{i_{1} \neq i_{2}=1}^{Z_{n-k}} Z_{k, i_{1}}^{(n-k)} Z_{k, i_{2}}^{(n-k)}}{\left(\sum_{i=1}^{Z_{n-k}} Z_{k, i}^{(n-k)}\right)\left(\sum_{i=1}^{Z_{n-k}} Z_{k, i}^{(n-k)}-1\right)} \mathbf{1}\left(\sum_{i=1}^{Z_{n-k}} Z_{k, i}^{(n-k)} \geq 2\right)\right) \\
& =\mathrm{E}\left(\frac{\sum_{i_{1} \neq i_{2}=1}^{Z_{n-k}} Z_{k, i_{1}}^{(n-k)} Z_{k, i_{2}}^{(n-k)}}{\left(\sum_{i=1}^{Z_{n-k}} Z_{k, i}^{(n-k)}\right)\left(\sum_{i=1}^{Z_{n-k}} Z_{k, i}^{(n-k)}-1\right)} \mathbf{1}\left(\sum_{i=1}^{Z_{n-k}} Z_{k, i}^{(n-k)} \geq 2\right) \mid Z_{n-k}>0\right) \mathrm{P}\left(Z_{n-k>0}\right) \\
& =\mathrm{E}\left(\phi_{k}\left(Z_{n-k}\right) \mid Z_{n-k}>0\right) \mathrm{P}\left(Z_{n-k}>0\right),
\end{aligned}
$$

where, for $j \geq 1$,

$$
\phi_{k}(j)=\mathrm{E}\left(\frac{\sum_{i_{1} \neq i_{2}=1}^{j} Z_{k, i_{1}} Z_{k, i_{2}}}{\left(\sum_{i=1}^{j} Z_{k, i}\right)\left(\sum_{i=1}^{j} Z_{k, i}-1\right)} \mathbf{1}\left(\sum_{i=1}^{j} Z_{k, i} \geq 2\right)\right)
$$


and $\left\{Z_{r, i}: r \geq 0\right\}, i=1,2, \ldots, j$, are i.i.d. copies of a Galton-Watson branching process with $Z_{0}=1$ and offspring distribution $\left\{p_{i}\right\}_{i \geq 0}$ satisfying the hypothesis of Theorem 2.2.

Similarly,

$$
c_{n}=\mathrm{E}\left(\psi_{k}\left(Z_{n-k}\right) \mid Z_{n-k}>0\right) \mathrm{P}\left(Z_{n-k}>0\right),
$$

where, for $j \geq 1, \psi_{k}(j)=\mathrm{E}\left(\mathbf{1}\left(\sum_{i=1}^{j} Z_{k, i} \geq 2\right)\right)$.

By Theorem 3.4, for any $Z_{0}$ with $\mathrm{E} Z_{0}<\infty$, and $k<\infty$,

$$
Z_{n-k} \mid Z_{n-k}>0 \stackrel{\mathrm{D}}{\rightarrow}\left\{b_{j}\right\}_{j \geq 1} \quad \text { as } n \rightarrow \infty,
$$

where $\left\{b_{j}\right\}_{j \geq 1}$ is as in Theorem 3.3(i). Thus, for each fixed $k \geq 1$,

$$
\mathrm{P}\left(n-X_{n}>k \mid Z_{n} \geq 2\right) \rightarrow \frac{\mathrm{E} \phi_{k}(Y)}{\mathrm{E} \psi_{k}(Y)} \equiv \pi_{k}
$$

where $Y$ is a random variable with distribution $\mathrm{P}(Y=j)=b_{j}, j \geq 1$.

It remains to show that if $\sum_{j=1}^{\infty} j \log j p_{j}<\infty$ then $\lim _{k \uparrow \infty} \pi_{k}=0$.

For $1 \leq j<\infty$,

$$
\begin{aligned}
\phi_{k}(j) & \leq \mathrm{P}\left(\text { there exist } i_{1}, i_{2}, i_{1} \neq i_{2}, 1 \leq i_{1}, i_{2} \leq j, Z_{k, i_{1}}>0, Z_{k, i_{2}}>0\right) \\
& \leq 1-\left(f_{k}(0)\right)^{j}-j\left(f_{k}(0)\right)^{j-1}\left(1-f_{k}(0)\right)
\end{aligned}
$$

and

$$
\psi_{k}(j)=\mathrm{P}\left(\sum_{i=1}^{j} Z_{k, i} \geq 2\right)=\mathrm{P}\left(\sum_{i=1}^{j} Z_{k, i} \geq 2 \mid \sum_{i=1}^{j} Z_{k, i} \geq 1\right) \mathrm{P}\left(\sum_{i=1}^{j} Z_{k, i} \geq 1\right) .
$$

So,

$$
\mathrm{E} \phi_{k}(Y)=\mathrm{E}\left(1-\left(f_{k}(0)\right)^{Y}-Y\left(f_{k}(0)\right)^{Y-1}\left(1-f_{k}(0)\right)\right)
$$

and

$$
\mathrm{E} \psi_{k}(Y)=\mathrm{E}\left(1-\left(f_{k}(0)\right)^{Y}\right) \mathrm{P}\left(\sum_{i=1}^{Y} Z_{k, i} \geq 2 \mid \sum_{i=1}^{Y} Z_{k, i} \geq 1\right) .
$$

Since $\sum_{j=1}^{\infty} j \log j p_{j}<\infty$ implies that $\mathrm{E} Y<\infty$, by Theorem 3.4(i),

$$
\lim _{k} \mathrm{P}\left(\sum_{i=1}^{Y} Z_{k, i} \geq 2 \mid \sum_{i=1}^{Y} Z_{k, i} \geq 1\right)
$$

exists and equals $1-b_{1}, 0<b_{1}<1$.

Next,

$$
\begin{aligned}
\frac{\mathrm{E} \phi_{k}(Y)}{\mathrm{E} \psi_{k}(Y)} \leq & \frac{\mathrm{E}\left(1-\left(f_{k}(0)\right)^{Y}\right)}{\mathrm{E}\left(1-\left(f_{k}(0)\right)^{Y}\right)}\left(1-\frac{\left(1-f_{k}(0)\right) \mathrm{E}\left(Y\left(f_{k}(0)\right)^{Y-1}\right)}{\mathrm{E}\left(1-\left(f_{k}(0)\right)^{Y}\right)}\right) \\
& \times \frac{1}{\mathrm{P}\left(\sum_{i=1}^{Y} Z_{k, i} \geq 2 \mid \sum_{i=1}^{Y} Z_{k, i} \geq 1\right)}
\end{aligned}
$$


By the monotone convergence theorem, as $k \rightarrow \infty$,

$$
\mathrm{E}\left(Y\left(f_{k}(0)\right)^{Y-1}\right) \rightarrow \mathrm{E} Y
$$

and

$$
\mathrm{E}\left(\frac{1-\left(f_{k}(0)\right)^{Y}}{1-f_{k}(0)}\right) \rightarrow \mathrm{E} Y .
$$

By Theorem 3.3(ii), $\sum_{j=1}^{\infty} j \log j p_{j}<\infty$ implies that $0<\mathrm{E} Y<\infty$ and this in turn implies, by Theorem 3.4(i),

$$
\lim _{k \rightarrow \infty} \mathrm{P}\left(\sum_{i=1}^{Y} Z_{k, i} \geq 2 \mid \sum_{i=1}^{Y} Z_{k, i} \geq 1\right)=1-b_{1}, \quad 0<b_{1}<1 .
$$

Thus,

$$
\varlimsup_{k \rightarrow \infty} \frac{\mathrm{E} \phi_{k}(Y)}{\mathrm{E} \psi_{k}(Y)} \leq \frac{1}{1-b_{1}}\left(1-\frac{\mathrm{E} Y}{\mathrm{E} Y}\right)=0,
$$

i.e. $\lim _{k \rightarrow \infty} \pi_{k}=0$, completing the proof of Theorem 2.2(ii).

(ii) Clearly,

$$
\mathrm{P}\left(n-Y_{n}>k \mid Z_{n} \geq 1\right)=\mathrm{P}\left(Y_{n}<n-k \mid Z_{n} \geq 1\right)=\frac{\mathrm{P}\left(\left(Y_{n}<n-k\right) \cap\left(Z_{n} \geq 1\right)\right)}{\mathrm{P}\left(Z_{n} \geq 1\right)} .
$$

The numerator is

$$
\begin{aligned}
& \mathrm{P}\left(\text { there exist } i_{1}, i_{2}, i_{1} \neq i_{2}, 1 \leq i_{1}, i_{2} \leq Z_{n-k} \ni Z_{k, i_{1}}^{(n-k)}>0, Z_{k, i_{2}}^{(n-k)}>0\right) \\
& \quad=\mathrm{E}\left(1-\left(f_{k}(0)\right)^{Z_{n-k}}-Z_{n-k}\left(f_{k}(0)\right)^{Z_{n-k}-1}\left(1-f_{k}(0)\right) ; Z_{n-k}>0\right) \\
& \quad=\mathrm{E}\left(1-\left(f_{k}(0)\right)^{Z_{n-k}}-Z_{n-k}\left(f_{k}(0)\right)^{Z_{n-k}-1}\left(1-f_{k}(0)\right) \mid Z_{n-k}>0\right) \mathrm{P}\left(Z_{n-k}>0\right) .
\end{aligned}
$$

For fixed $k \geq 1$, the first term above goes to, by Theorem 3.3(i),

$$
\mathrm{E}\left(1-\left(f_{k}(0)\right)^{Y}-Y\left(f_{k}(0)\right)^{Y-1}\left(1-f_{k}(0)\right)\right) .
$$

Also, by Theorem 3.3(iii), for fixed $k \geq 1$,

$$
\frac{\mathrm{P}\left(Z_{n}>0\right)}{\mathrm{P}\left(Z_{n-k}>0\right)} \rightarrow m^{k} \quad \text { as } n \rightarrow \infty .
$$

Thus, for fixed $k \geq 1$,

$$
\lim _{n \rightarrow \infty} \mathrm{P}\left(n-Y_{n}>k \mid Z_{n}>0\right)=\frac{\mathrm{E}\left(1-\left(f_{k}(0)\right)^{Y}-Y\left(f_{k}(0)\right)^{Y-1}\left(1-f_{k}(0)\right)\right)}{m^{k}} \equiv \tilde{\pi}_{k} .
$$

Now,

$$
\tilde{\pi}_{k}=\frac{\mathrm{E}\left(1-\left(f_{k}(0)\right)^{Y}\right)}{m^{k}}\left(1-\frac{\left(\mathrm{E} Y\left(f_{k}(0)\right)^{Y-1}\right)\left(1-f_{k}(0)\right)}{\mathrm{E}\left(1-\left(f_{k}(0)\right)^{Y}\right)}\right) .
$$


As argued in the proof of (i) above, under the hypothesis that $\sum_{j=1}^{\infty} j \log j p_{j}<\infty$,

$$
\mathrm{E}\left(\frac{1-\left(f_{k}(0)\right)^{Y}}{1-f_{k}(0)}\right) \rightarrow 1<\mathrm{E} Y<\infty, \quad \mathrm{E} Y\left(f_{k}(0)\right)^{Y-1} \rightarrow 1<\mathrm{E} Y<\infty
$$

Also,

$$
\mathrm{E}\left(\frac{1-\left(f_{k}(0)\right)^{Y}}{m^{k}}\right)=\frac{1-f_{k}(0)}{m^{k}} \mathrm{E}\left(\sum_{j=0}^{Y-1}\left(f_{k}(0)\right)^{j}\right)
$$

and, by Theorem 3.3(iii),

$$
\lim _{k \rightarrow \infty} \frac{1-f_{k}(0)}{m^{k}}=\frac{1}{\mathrm{E} Y} .
$$

Thus, $\lim _{k \rightarrow \infty} \tilde{\pi}_{k}=0$. This completes the proof of Theorem 2.2(ii).

\section{Acknowledgement}

The author would like to convey his grateful thanks to the anonymous referee for the suggestions given in Remarks 2.3 and 2.4 of using the continuum random tree for Theorem 2.1, the results of Geiger [3] for Theorem 2.2, and also the relevance of the results on Itô's excursion theory due to Le Gall [5].

\section{References}

[1] Athreya, K. B. (2012). Coalescence in the recent past in rapidly growing populations. Stoch. Process. Appl. 122, 3757-3766.

[2] Athreya, K. B. And Ney, P. E. (2004). Branching Processes. Dover, Mineola, NY.

[3] GeIger, J. (1999). Elementary new proofs of classical limit theorems for Galton-Watson processes. J. Appl. Prob. 36, 301-309.

[4] Kallenberg, O. (1986). Random Measures, 4th edn. Academic Press, London.

[5] Le Gall, J.-F. (2010). Itô's excursion theory and random trees. Stoch. Process. Appl. 120, 712-749.

[6] Zubkov, A. M. (1975). Limit distribution of the distance to the nearest common ancestor. Theory Prob. Appl. 20, 602-612. 